\title{
In silico search for planar hexacoordinate Silicon atom: A kinetically viable species
}

\author{
Amlan Kalita ${ }^{1}$, Shahnaz Rohman ${ }^{1}$, Chayanika Kashyap ${ }^{1}$, Sabnam Ullah$^{1}$, Indrani Baruah ${ }^{1}$, \\ Lakhya Mazumder ${ }^{1}$, and Ankur Guha ${ }^{1}$ \\ ${ }^{1}$ Cotton University
}

January 23, 2021

\begin{abstract}
In silico search for planar hexacoordinate silicon center has been initiated by global minimum screening with density functional theory and energy refinement using coupled cluster theory. The search resulted in a local minimum of SiAl3Mg3H2+ structure which contains a planar hexacoordinate silicon center ( $\mathrm{phSi}$ ). The phSi structure is $5.8 \mathrm{kcal} / \mathrm{mol}$ higher in energy than the global minimum. However, kinetic studies reveal that the local minimum structure has enough stability to be detected experimentally. Born-Oppenheimer molecular dynamics (BOMD) simulations reveal that the phSi structure can be maintained up to $400 \mathrm{~K}$. The formation of multiple bonds between the central silicon atom and framework aluminium atom is the key stabilizing factor for the planar structure.
\end{abstract}

\section{Hosted file}

MS-IJQC.pdf available at https://authorea.com/users/297273/articles/505383-in-silico-searchfor-planar-hexacoordinate-silicon-atom-a-kinetically-viable-species 